Proceedings of the 45th International School and Conference on the Physics of Semiconductors "Jaszowiec" 2016, Szczyrk

\title{
Landau Levels of Double-Weyl Nodes in a Simple Lattice Model
}

\author{
R. RECHCIŃSKI ${ }^{a, b, *}$ AND J. TWORZYDŁO ${ }^{a}$ \\ ${ }^{a}$ Faculty of Physics, University of Warsaw, L. Pasteura 5, 02-094 Warszawa, Poland \\ ${ }^{b}$ Institute of Physics, Polish Academy of Sciences, Aleja Lotników 32/46, PL-02668 Warsaw, Poland
}

\begin{abstract}
In the Weyl semimetals, a recently discovered class of bulk materials, inverted band gap closes in the first Brillouin zone at topologically protected points of degeneracy called the Weyl nodes. By using the Chern number formalism it is possible to assign to each of the nodes an integer topological charge $Q$. While around typical Weyl points the energy disperses linearly in all three directions, double-Weyl nodes (with $|Q|=2$ ) exhibit quadratic dispersion in two directions and linear in the third one. We use a simple 2-band tight-binding lattice model to investigate the dispersion of the Landau levels in the presence of quantizing magnetic field in the vicinity of a double-Weyl node. In the long wavelength limit we obtain analytically the expected presence of two chiral levels. In addition, we find numerous level crossings between the non-chiral Landau levels and the chiral ones, a feature which is distinct from the single node case. Calculations for a finite-size sample, both with periodic and hard-wall boundary conditions (the latter corresponding to slab geometry), show that the two chiral levels hybridize in the conduction band with the two lowest non-chiral Landau levels. In the case of slab geometry these four levels are responsible for the formation of a protected surface state.
\end{abstract}

DOI: 10.12693/APhysPolA.130.1179

PACS/topics: 71.70.Di, 73.43.-f

\section{Introduction}

Theoretical interest in topological semimetals dates back to early 80 s, where such a system was introduced in the context of high-energy physics [1]. The low energy theory of a topological semimetal describes two valleys in the band structure, each corresponding to a Weyl fermion. One may envision such a system as the 3D generalization of well-known two Dirac electron valleys in graphene. General arguments guarantee [1] the existence of at least two valleys of the Weyl fermions in any lattice representation of this system. The nodal points, where the gap closes, are topologically protected from gapping under any smooth perturbation of the lattice model. The quantifying characteristic of a single node topology is the Chern number $|Q|=1$, calculated as an integral of the Berry curvature on a small sphere containing the node.

The recent renewal of interest in topological semimetals has begun with the identification [2] of physically measurable consequences of topological nature of the nodal states. The so-called chiral anomaly arises when the Weyl fermions are placed in the external magnetic field. Although a full theory of quantum transport $[3,4]$ due to the chiral anomaly is still under development, one can form a basic physical intuition by considering the Landau levels of the Weyl fermions. The characteristic 0th Landau level develops when the external magnetic field is applied along the axis connecting the nodes $\left(k_{z}\right.$ in our notation). This level is called chiral as it is characterized by a constant group velocity in the vicinity of a single node. The net physical current in a crystal is conserved,

*corresponding author; e-mail: rafal.rechcinski@ifpan.edu.pl as the group velocity of the 0th Landau level has opposite signs for the two nodes.

The purpose of the present contribution is to investigate the Landau level structure in a model of topological semimetal with a higher topological charge $Q=2$. Topological semimetals with this property has been proposed theoretically $[5,6]$ and by now some realistic systems are considered [7]. Here we limit ourselves to a simple lattice formulation, which is equivalent to the one proposed in Ref. [6]. To our knowledge the Landau level quantization in such a model has not been considered so far.

\section{The model}

We consider a simple 2-band tight-binding Hamiltonian, which is expressed in the diagonal form in the crystal momenta as follows:

$$
\begin{aligned}
& \quad H(\boldsymbol{k})=\boldsymbol{h}(\boldsymbol{k}) \cdot \boldsymbol{\sigma}, \\
& \text { with } \\
& \qquad \begin{array}{l}
h_{x}=2 t^{\prime}\left(\cos k_{y}-\cos k_{x}\right), \\
h_{y}=t^{\prime} \sin k_{x} \sin k_{y}, \\
h_{z}=\mu-t \cos k_{x}-t \cos k_{y}-2 \tilde{t} \cos k_{z},
\end{array}
\end{aligned}
$$

where $t^{\prime}, t$, and $\tilde{t}$ are the hopping parameters and $\mu$ is half the energy difference between levels on a single lattice site. The energy dispersion relations of the conduction (denoted by + ) and valence $(-)$ bands are given by $E_{ \pm}= \pm|\boldsymbol{h}(\boldsymbol{k})|$, which follows from the fact that $H^{2}(\boldsymbol{k})=\boldsymbol{h}^{2}(\boldsymbol{k})$ due to the anticommutation relations of the Pauli matrices $\left\{\sigma_{i}, \sigma_{j}\right\}=2 \delta_{i j}$. If the inequality $|2 t-2 \tilde{t}|<\mu<2 \tilde{t}+2 t$ is satisfied the bands are degenerate at exactly two points in the first Brillouin zone, which we denote by $\boldsymbol{k}_{\mathrm{W}}=\left(0,0, \pm \arccos \frac{\mu-2 t}{2 \tilde{t}}\right)$. Around these points the bands disperse linearly along the $z$ axis with $\partial E_{+} / \partial k_{z}=\sqrt{(2 \tilde{t})^{2}-(\mu-2 t)^{2}}$ and quadratically 
in the $x$ and $y$ directions with $\partial^{2} E_{+} / \partial k_{x}^{2}=\partial^{2} E_{+} / \partial k_{y}^{2}=$ $\sqrt{t^{2}+4 t^{\prime 2}}$.

To show that these band crossings are double-Weyl nodes we calculate the Chern number $Q$ on small spheres enclosing each of the degeneracy points in the $\boldsymbol{k}$ space. Let us first note that in the case of 2-band models it is easier to calculate $Q$ in the $\boldsymbol{h}$ space, that is in the threedimensional space of traceless Hermitian $2 \times 2$ matrices. The Berry curvature is defined by

$$
\boldsymbol{\Omega}^{(n)}(\boldsymbol{k})=\mathrm{i} \nabla_{\boldsymbol{k}} \times\left\langle u_{n}(\boldsymbol{k})\left|\nabla_{\boldsymbol{k}}\right| u_{n}(\boldsymbol{k})\right\rangle,
$$

where $u_{n}(\boldsymbol{k})$ denotes the wave function of two bands $n= \pm$. We can express the Berry curvature in the $\boldsymbol{h}$ space, obtaining a simple inverse-square form

$$
\boldsymbol{\Omega}^{( \pm)}(\boldsymbol{h})=\mp \frac{\boldsymbol{h}}{2|\boldsymbol{h}|^{3}} .
$$

The integral over a sphere $S=\left\{\boldsymbol{k}:\left|\boldsymbol{k}-\boldsymbol{k}_{\mathrm{W}}\right|=k_{S}\right\}$ :

$$
Q^{(n)}=-\frac{1}{2 \pi} \oint_{S} \boldsymbol{\Omega}^{(n)}(\boldsymbol{k}) \mathrm{d} S,
$$

which defines the Chern number, is now easily evaluated by changing variables according to Eq. (2), since the form of the integrand (4) enables us to use the Gauss flux theorem. To obtain the integer value of $Q^{(n)}$ it is therefore enough to consider how many times and in which direction the parametric surface $S_{h}=\left\{\boldsymbol{h}(\boldsymbol{k}):\left|\boldsymbol{k}-\boldsymbol{k}_{\mathrm{W}}\right|=k_{S}\right\}$ winds over the monopole at $\boldsymbol{h}=0$.

Expanded around $\pm \boldsymbol{k}_{\mathrm{W}}$ the Hamiltonian becomes

$$
\begin{aligned}
& h_{x} \approx t^{\prime}\left(k_{x}^{2}-k_{y}^{2}\right), \\
& h_{y} \approx t^{\prime} k_{x} k_{y}, \\
& h_{z} \approx \pm \sqrt{(2 \tilde{t})^{2}-(\mu-2 t)^{2}}\left(k_{z} \mp k_{z \mathrm{~W}}\right) .
\end{aligned}
$$

If we introduce spherical coordinates $(k, \theta, \phi)$ around the Weyl node $\pm \boldsymbol{k}_{\mathrm{W}}$ :

$$
k_{z} \mp k_{z \mathrm{~W}}=k \cos \theta, \quad k_{x} \pm \mathrm{i} k_{y}=k \sin \theta \mathrm{e}^{ \pm \mathrm{i} \phi},
$$

we get the parametric surface

$$
\begin{aligned}
& h_{x}=t^{\prime} k^{2} \sin ^{2} \theta \cos (2 \phi), \\
& h_{y}=t^{\prime} k^{2} \sin ^{2} \theta \sin (2 \phi), \\
& h_{z}= \pm \sqrt{(2 \tilde{t})^{2}-(\mu-2 t)^{2}} k \cos \theta,
\end{aligned}
$$

which clearly winds twice over $\boldsymbol{h}=0$ in opposite directions for each of the points.

By a direct calculation we have confirmed that the Chern number at the $k_{z}=0$ plane is $|Q|=2$ while the $k_{z}=\pi$ plane has $Q=0$. Thus we can conclude that in a slab truncated along [100] plane the topologically protected surface states should appear between $-k_{z \mathrm{~W}}$ and $+k_{z \mathrm{~W}}$.

\section{Landau levels}

We use two methods to calculate the Landau levels in the magnetic field parallel to the $k_{z}$ axis, that is the axis on which the double-Weyl nodes appear. In the first method we work with the continuous model in the lowest non-zero order of the Taylor expansion around the $k_{z}$ axis and introduce the usual raising and lowering of the Landau level operators. The second method incorporates the Peierls substitition on a lattice model with either periodic or hard-wall boundary conditions.

For the analytical derivation we pick the vector potential in the axial gauge $\boldsymbol{A}(\boldsymbol{r})=(-B y / 2, B x / 2,0)$ and substitute the electron quasimomentum

$$
\boldsymbol{k} \rightarrow \boldsymbol{k}^{\prime}=\boldsymbol{k}+\boldsymbol{A},
$$

where we put $|e|=1$ and $\hbar=1$. Since

$$
\left[k_{y}^{\prime}, k_{x}^{\prime}\right]=\mathrm{i} B
$$

which resembles the canonical commutation relation $[x, p]=i \hbar$, we are able to introduce the raising and lowering operators in an analogy to a quantum harmonic oscillator

$$
a=\frac{k_{x}^{\prime}-\mathrm{i} k_{y}^{\prime}}{\sqrt{2 B}}, \quad a^{\dagger}=\frac{k_{x}^{\prime}+\mathrm{i} k_{y}^{\prime}}{\sqrt{2 B}}
$$

(without loss of generality we pick $B>0$ ). Our Hamiltonian (2) can be expanded around the $k_{z}$ axis, which gives

$$
h_{z} \approx \mu-2 t+\frac{t}{2}\left(k_{x}^{2}+k_{y}^{2}\right)-2 \tilde{t} \cos k_{z},
$$

with $h_{x}$ and $h_{y}$ the same as in Eq. (6). After substitution (9) the Hamiltonian expressed with the operators (11) becomes

$$
\begin{aligned}
& H\left(k_{z}\right)=2 t^{\prime} B\left(a^{2} \sigma_{+}+a^{\dagger^{2}} \sigma_{-}\right) \\
& \quad+t B\left(a^{\dagger} a+\frac{1}{2}\right) \sigma_{z}+f\left(k_{z}\right) \sigma_{z},
\end{aligned}
$$

where $\sigma_{ \pm}=\frac{1}{2}\left(\sigma_{x} \pm i \sigma_{y}\right)$ and $f\left(k_{z}\right)=\mu-2 t-2 \tilde{t} \cos k_{z}$. Eigenstates of the Hamiltonian can be expressed in the basis $\{|n, \pm\rangle\}$ where $n=0,1,2, \ldots$ is the Landau level of a free particle and \pm denotes the eigenstate of $\sigma_{z}$. By a straightforward calculation we obtain that $|0,-\rangle$ and $|1,-\rangle$ are eigenstates with dispersions

$$
\begin{aligned}
& E_{0}\left(k_{z}\right)=-\frac{t B}{2}-f\left(k_{z}\right), \\
& E_{1}\left(k_{z}\right)=-\frac{3 t B}{2}-f\left(k_{z}\right) .
\end{aligned}
$$

These are the peculiar chiral Landau levels. Other eigenvalues can be calculated by making use of the fact that the Hamiltonian splits into blocks spanned by the states $\{|n-2,+\rangle,|n,-\rangle\}$ (for $n=2,3,4, \ldots$ ), which can be diagonalized to yield pairs of Landau levels

$$
\begin{aligned}
& E_{n}^{ \pm}\left(k_{z}\right)=-t B \\
& \quad \pm \sqrt{\left[t B\left(n-\frac{1}{2}\right)+f\left(k_{z}\right)\right]^{2}+4 t^{\prime 2} B^{2} n(n-1) .}
\end{aligned}
$$

The Landau levels obtained in this way are plotted in Fig. 1 on the left hand side.

For the second method we discretize our tight binding Hamiltonian in the $x$ direction (putting a unit lattice constant) to get

$$
\begin{aligned}
H & =U \sum_{x}|x\rangle\left\langle x\left|+T \sum_{x}\right| x-1\right\rangle\langle x| \\
& +T^{\dagger} \sum_{x}|x+1\rangle\langle x|,
\end{aligned}
$$


where

$$
\begin{gathered}
U=\left(\begin{array}{cc}
\mu-t \cos k_{y}-2 \tilde{t} \cos k_{z} & 2 t^{\prime} \cos k_{y} \\
2 t^{\prime} \cos k_{y} & -\mu+t \cos k_{y}+2 \tilde{t} \cos k_{z}
\end{array}\right), \\
T=\left(\begin{array}{cc}
-\frac{t}{2} & t^{\prime}\left(-1-\frac{1}{2} \sin k_{y}\right) \\
t^{\prime}\left(-1+\frac{1}{2} \sin k_{y}\right) & \frac{t}{2}
\end{array}\right),
\end{gathered}
$$

and $x$ denotes lattice sites labeling (100) surfaces. This time we work in the Landau gauge with $\boldsymbol{A}(\boldsymbol{r})=$ $(0, B x, 0)$. The Peierls substitution applied to such a Hamiltonian reduces to substituting $k_{y} \rightarrow k_{y}+B x$ in $U$ matrices belonging to the node at the position $x$ and $k_{y} \rightarrow k_{y}+B(x+1 / 2)$ in $T$ matrices describing the hopping between $x$-th and $(x+1)$-th nodes.

In order to reproduce results obtained in the continuous model we consider a finite crystal of length $N_{x}$ with periodic boundary conditions (PBC), so that $\left\langle N_{x}-1|H| 0\right\rangle=T,\left\langle 0|H| N_{x}-1\right\rangle=T^{\dagger}$ (lattice sites are numbered $\left.x=0,1, \ldots, N_{x}-1\right)$. The external magnetic field has to be commensurate with the sample's length and may assume only a discrete set of values $B=2 m \pi / N_{x}$ with $m \in \mathbb{Z}$.

The Hamiltonian (16) with $\left\langle N_{x}-1|H| 0\right\rangle=$ $\left\langle 0|H| N_{x}-1\right\rangle=0$, that is with hard-wall boundary conditions applied, will serve us as a model of doubleWeyl semimetal truncated in the $x$ direction (slab geometry). It should be noted that in this case the Landau levels are not degenerate in $k_{y}$, contrary to the case of crystal with PBC.

In Fig. 1 we plot the comparison of results of the Landau levels dispersions along the $k_{z}$ axis for the continuous model, the discrete model with $\mathrm{PBC}$ and the latter with hard-wall BC. The plots show only the vicinity of a single double-Weyl node. All figures are plotted with the parameters $\mu=2, t^{\prime}=0.8, t=1, \tilde{t}=0.4$, sample width $N_{x}=500$, and magnetic field $B=2 \pi / 500$. This results in the magnetic length $\ell_{B}=1 / \sqrt{B} \approx 9$ lattice constants, which is $c a$. 50 times lower than the slab's width. Therefore the finite size effects should be less significant than the Landau quantization.

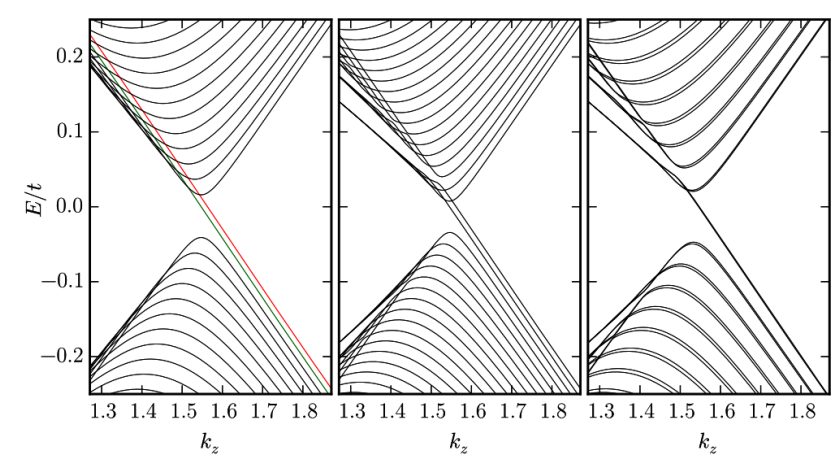

Fig. 1. Landau level dispersions along the $k_{z}$ axis. Left to right: analytical calculations in the long wavelength limit (colored lines are the chiral levels), calculations on a lattice with $\mathrm{PBC}$, calculations on a lattice with hardwall BC.
Results in the long wavelength limit show the presence of two topologically protected chiral Landau levels. These and other dispersion lines cross with each other numerous times between $-k_{z \mathrm{~W}}$ and $k_{z \mathrm{~W}}$, that is in the topologically nontrivial region of the Brillouin zone. Exact numerical results for a translationally invariant, but finite sample (with PBC) depart significantly from the long wavelength limit analysis. The difference persists for a finer lattice and bigger sample, and also in a wide range of the model parameters. We find that the chiral levels first cross the two lowest Landau levels in the conduction band, and then hybridize with the next two levels by an avoided crossing. Under close inspection we find that this scenario repeats for the crossings with the four higher Landau levels, which can be seen in Fig. 2. Remarakably, the crossing-avoided crossing sequence is the same for Landau levels calculated in slab geometry, as illustrated in the third part in Fig. 1. The lowest state that forms between $-k_{z \mathrm{~W}}$ and $k_{z \mathrm{~W}}$ is the surface state, thus it has to be of topological origin. The exact connection between the hybridization of the lowest Landau levels and formation of the surface state in our case is not yet clear. However, it is a feature distinct from a single node $Q=1$ Weyl semimetal.

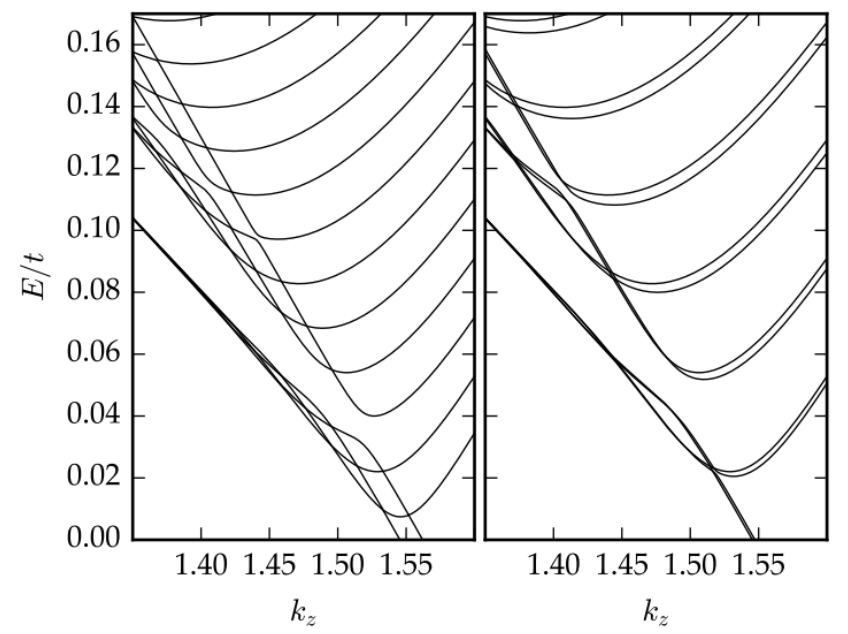

Fig. 2. Closed-up Landau level dispersions for a lattice with PBC (left-hand side) and hard-wall BC (righthand side), demonstrating the hybridization of four of the lowest Landau levels.

We conclude that the topological nature of the chiral states can have profound consequences when those states cross the other, non-chiral Landau levels. The interplay of full crossing-anticrossing may be used as an indicator of the presence of chiral states in future experiments e.g. optical spectra of a clean, thin slab of double-Weyl semimetal material.

\section{Acknowledgments}

The author (J.T.) is supported by a grant of the Polish National Science Center no. 2014/15/B/ST3/03833. 


\section{References}

[1] H.B. Nielsen, M. Ninomiya, Phys. Lett. B 130, 389 (1983).

[2] A.A. Zyuzin, A.A. Burkov, Phys. Rev. B 86, 115133 (2012).

[3] S. Zhong, J.E. Moore, I. Souza, Phys. Rev. Lett. 116, 077201 (2016).

[4] P. Baireuther, J.A. Hutasoit, J. Tworzydło, C.W.J. Beenakker, New J. Phys. 18, 045009 (2016).
[5] C. Fang, M. Gilbert, X. Dai, B.A. Bernevig, Phys. Rev. Lett. 108, 266802 (2012).

[6] G. Xu, H. Weng, Z. Wang, X. Dai, Z. Fang, Phys. Rev. Lett. 107, 186806 (2011).

[7] S.-M. Huang, S.Y. Xu, I. Belopolski, C.C. Lee, G. Chang, B. Wang, N. Alidoust, M. Neupane, H. Zheng, D. Sanchez, A. Bansil, G. Bian, H. Lin, M.Z. Hasan, Proc. Natl. Acad. Sci. USA 113, 1180 (2016). 